\title{
Natural history of pellets inside human body, a prospective observational study
}

\author{
Azher Mushtaq ${ }^{*}$, Zahid Mohammad ${ }^{1}$, Malik Suhail Ahmad ${ }^{1}$, Mumtaz ud din Wani' ${ }^{2}$, Fahad ul Islam Mir $^{3}$ \\ ${ }^{1}$ Registrar Surgery, Govt. Medical College, SMHS, Srinagar, India \\ ${ }^{2}$ Professor of Surgery, Govt. Medical College SMHS, Srinagar, India \\ ${ }^{3}$ Junior Resident. Department of Surgery, SMHS Hospital, Srinagar, India
}

\section{Introduction}

In the modern world violence and trauma is emerging as a new public health problem. Violence either at individual like Suicide, Homicide or at a large scale like Communal riots, political suppression of communities etc is taking a heavy toll of human lives and is telling upon the health care of the communities. Kashmir being a conflict zone, violence particularily weapon violence is a routine happening in this region. Street protest and clashes form the major source of weapon violence. The security forces use different kinds of armamentarium like; tear gas, pawa shells, Pellet guns etc to quell the protestors.

The introduction of pellet gun as a non-lethal weapon for controlling rioting mobs has posed a serious challenge to the surgeons in treating pellet victims. Pellet gun is a form of short gun with a 20 inch barrel operating at low pressures as low as 50 atmospheres [1]. Pellets are small metallic particles of irregular shape housed in a nonmetallic cartridge (Figure 1). One cartridge contains hundreds of such metal pellets. After being fired from the pellet gun, a cartridge bursts and spreads hundreds of these metallic pellets in air at high velocities capable of penetrating soft tissues and even breaching the peritoneal cavity. The pellets being metallic persist in the human body unless removed.

There are not much studies available in literature on the management and long term follow up of pellet victims [2]. We therefore performed a study to know the long term physical effects of these metal pellets inside human body and the need for their removal from the body.

\section{Materials and methods}

The study was performed in a tertiary care hospital of Government Medical College Srinagar during an episode of civilian unrest in july 2016. The hospital is a sole tertiary care hospital of the Kashmir valley that received majority of injured patients during the turmoil of 2016. A total of 70 pellet hit patients with X- ray and CT scan documented pellets in parietal soft tissues were enrolled in our study. After initial stabilisation and resuscitation, the patients who were discharged uneventfully were followed for 1 year period. Only those patients who had no associated eyeball injury and who had not undergone any surgery were enrolled in our study. The follow up was carried at 1 month, 3 month, 6 month and at 1 year in all patients. On each follow up a routine general physical examination was carried out and any complaints by the patient associated with the pellets were ascertained. An x-ray was done on each follow up to know the status of pellets inside the body.

\section{Results and observations}

In a total of 70 patients, majority of patients in our study were in the age group of 20-29 years with a mean age of $26.5 \pm 8.9$ years. 61 were males and 9 patients were females (Figure 2).

Body parts predominantly affected by pellets among patients in our study is shown in the graph below (Figure 3).

Symptoms noted by the patients in our study are shown in the graph below (Figure 4).

\section{Discussion}

Pellet trauma is a rare form of weapon violence on which very few human studies are available in the literature. There are no definite protocols available for the short as well as long term management of such victims. In 2010 episode of violence, a total of 198 patients had suffered pellet injuries and the most common sites of injury were the extremities (47.9\%), abdomen (36.3\%) and chest (31.3\%) [3]. During another recent turmoil in the valley, our hospitals were again flooded with such pellet injured victims creating a serious challenge to the surgeons in managing such patients. In our study, majority of the pellet victims were in $20-29$ years age group. $87 \%$ being males. The mean age was $26.5 \pm 8.9$ years. In our study we included pellet injured patients who were not operated and who were not having any injury to eye globe. Because it would be difficult in the post-operative period to ascertain whether the pain was due to operative wound or due to pellets in the parietal wall. Immediately after the impact, the pellets penetrate the skin and get lodged in the soft tissues beneath the skin producing multiple puncture wounds on the body and severe pain. A classical pellet induced wound can be described as a small puncture wound 3-4 $\mathrm{mm}$ in diameter with marked surrounding erythema and blood oozing from the puncture site. In some cases, a pellet is seen peeping through the punctured skin defect (Figure 5). After an initial stabilisation and later uneventful discharge, the patients were followed regularly for 1 year. $64 \%$ of patients in our study had multiple pellets in torso (back, chest wall and abdominal wall); $42 \%$ had pellets in limbs and $10 \%$ had pellets in head and neck region (Figure 6).

Correspondence to: Azher Mushtaq, Registrar Surgery, Govt. Medical College, SMHS, Srinagar, India, Tel: 0194250 4114; E-mail: Azharmushtaq82@gmail.com

Received: September 27, 2017; Accepted: November 01, 2017; Published: November 04, 2017 

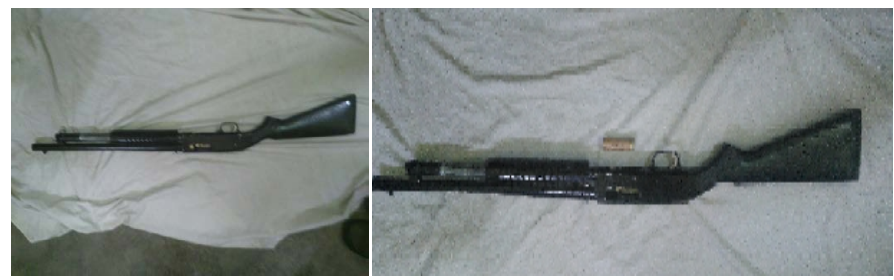

Figure 1. Pellet gun along with the catridge containing pellets.

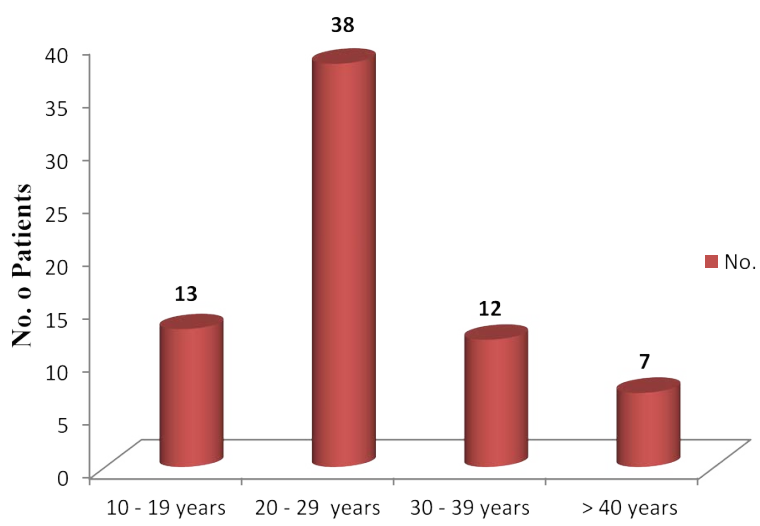

Figure 2. Age distribution of patients in our study.

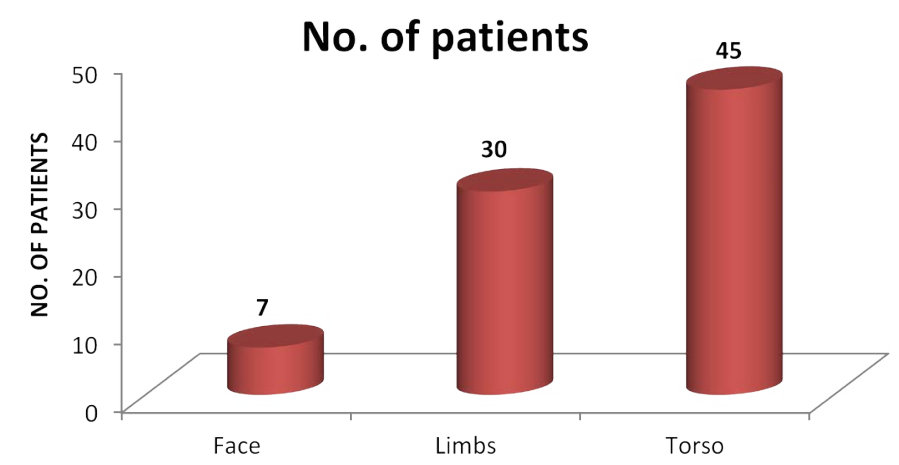

Figure 3. Sites involved by pellets in patients in our study. The total does not equal to total number of patients because many patients had involvement of multiple body parts.

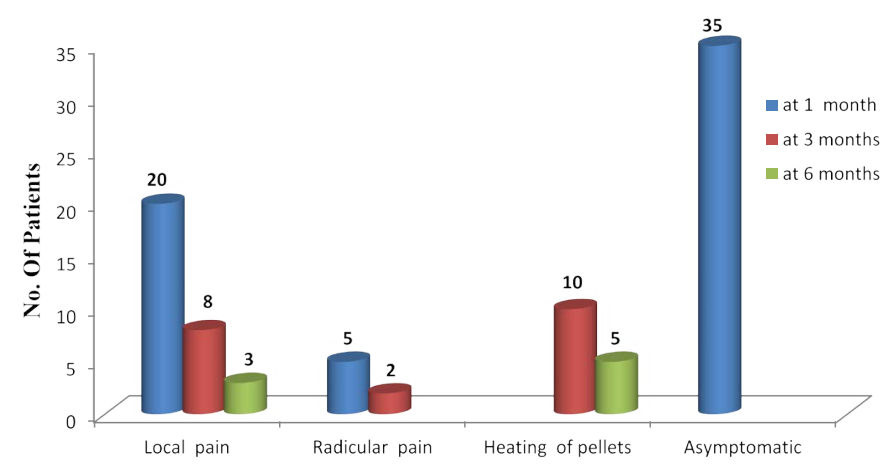

Figure 4. Predominant symptoms present among patients in our study at 1;2 and 3 months follow-up.

At 1 month follow-up 50\% (35/70 patients) had no specific complaints regarding the pellets inside their body while as $28 \%(20 / 70$ patients) complained of local pain in the region of pellets in their body. All the patients described the pain as mild in intensity and was not disturbing their day to day activities but some patients ( $8 / 20$ patients) described the pain to worsen during night and was disturbing their sleep. The most distressing symptom noted at 1 month follow up was the radicular type pain in upper arm radiating along the forerarm noted in 5 patients. All these patients were given a course of Methylcobalamine and gabapentin for one month and occasional oral diclofenac when needed.

At 3 months follow up 8 patients continued with mild local pain at the site of pellets. 3 of these patients had pellets in forehead region while as 5 patients had large number of pellets in the upper back. These patients were again put on one-month therapy with Methylcobalamine and gabapentin. 5 of these patients responded while as 3 patients with pellets in forehead region ended up in removal of these local pellets later. Among 5 patients with radicular type pain in forearm, 3 patients responded to Methylcobalamine and gabapentin regime, but two patients had their pellets removed because of severe disturbing pain in forearm in the distribution of ulnar nerve. Both these patients had few pellets on the medial aspect of elbow in the region of ulnar nerve which were removed, and the pain got relieved.

One of the very unusual complaints noted at around 3 months follow up was the feeling of hot sensation at the site of pellet aggregation in the body. The complaint became more frequent as the winter approached and the atmospheric temperature began to fall resulting in frequent use of Kangri by the people in our society. Kangri is a local term used for a clay pot containing burning coal (Figure 7) which is used in winters to keep a person warm. The complaint was more common and distressing in patients with a large aggregate of pellets localised at one place in anterior abdominal wall. 10 patients in our study had this distressing complaint at 3 months follow up. 5 of these patients lost the followup while as 5 patients got their pellets removed at 6 months follow up because of this complaint (Figure 8).

Even after 11 months, there was no change in the radiographic appearance of the pellets inside body (Figure 9). However, the external puncture wounds healed with small scars (Figure 10). None of the patients in our study after 1 year follow up had any complaints of Acute or chronic lead poisoining. So serum lead levels were not done in patients in our study.

\section{Conclusion}

From this study, we observed that the pellets inside human body become engulfed by the fibrous tissue and persist in the body without

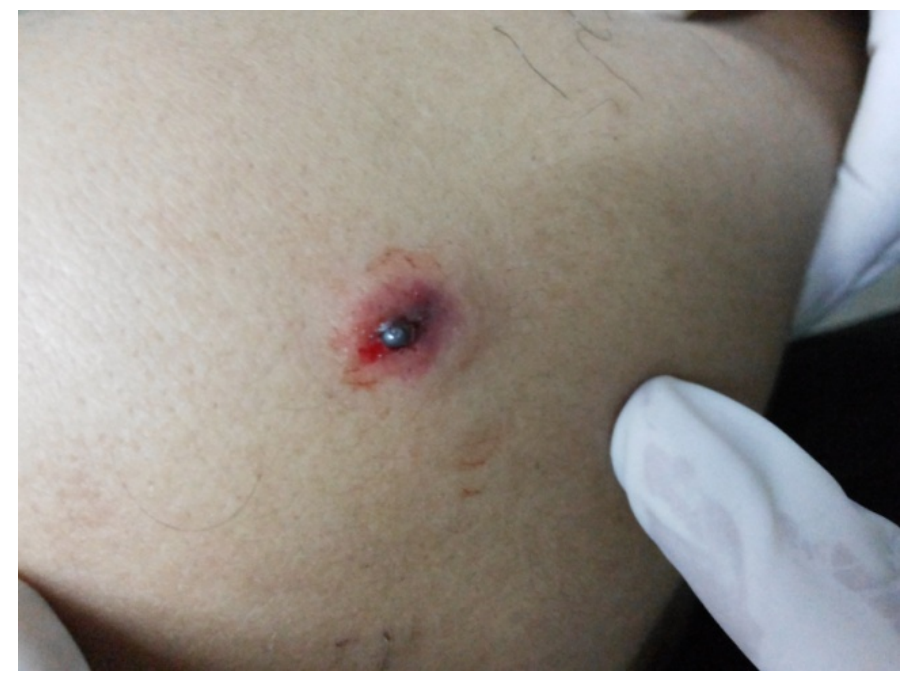

Figure 5. A classical pellet induced wound with a pellet peeping out through the puncture site. 


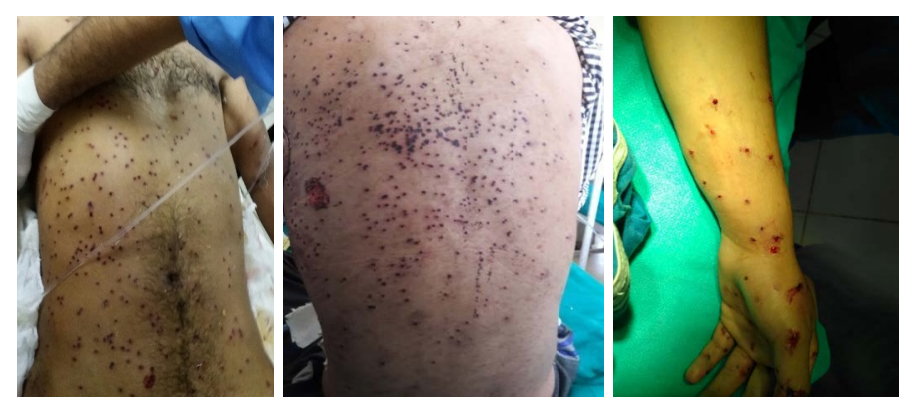

Figure 6. Pellets in anterior abdominal and chest wall (Right); Multiple pellets in back (middle); Pellets in forearm and hand (left).

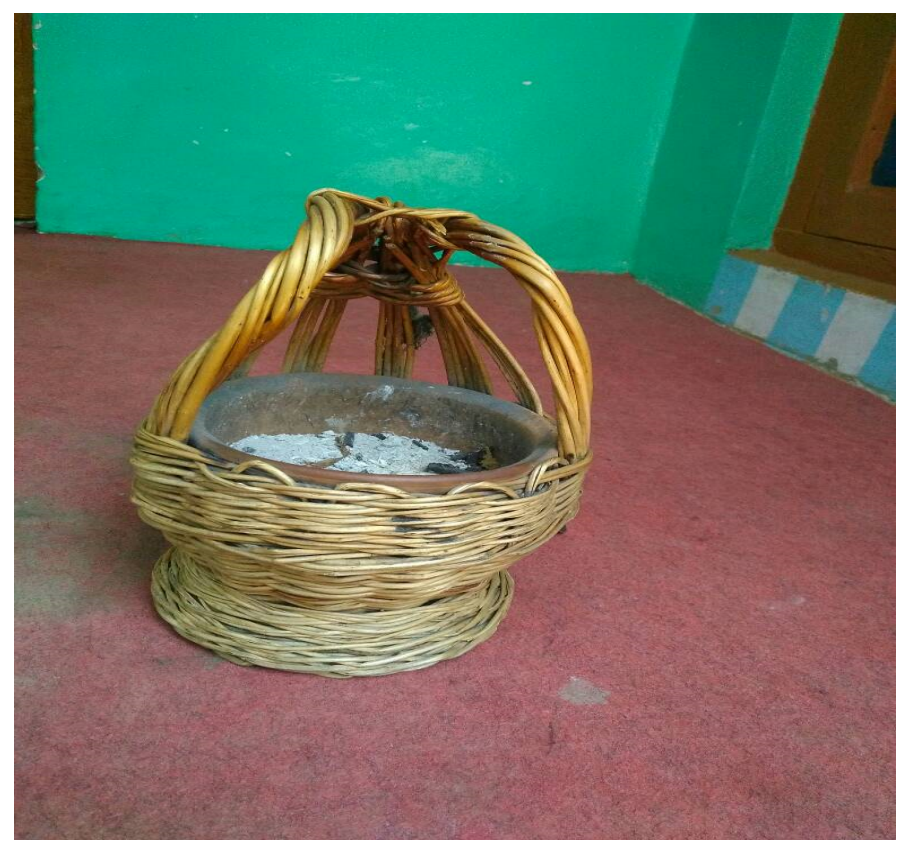

Figure 7. Kangri; A clay pot with wooden insulation containing burning coal to keep a person warm in winter.

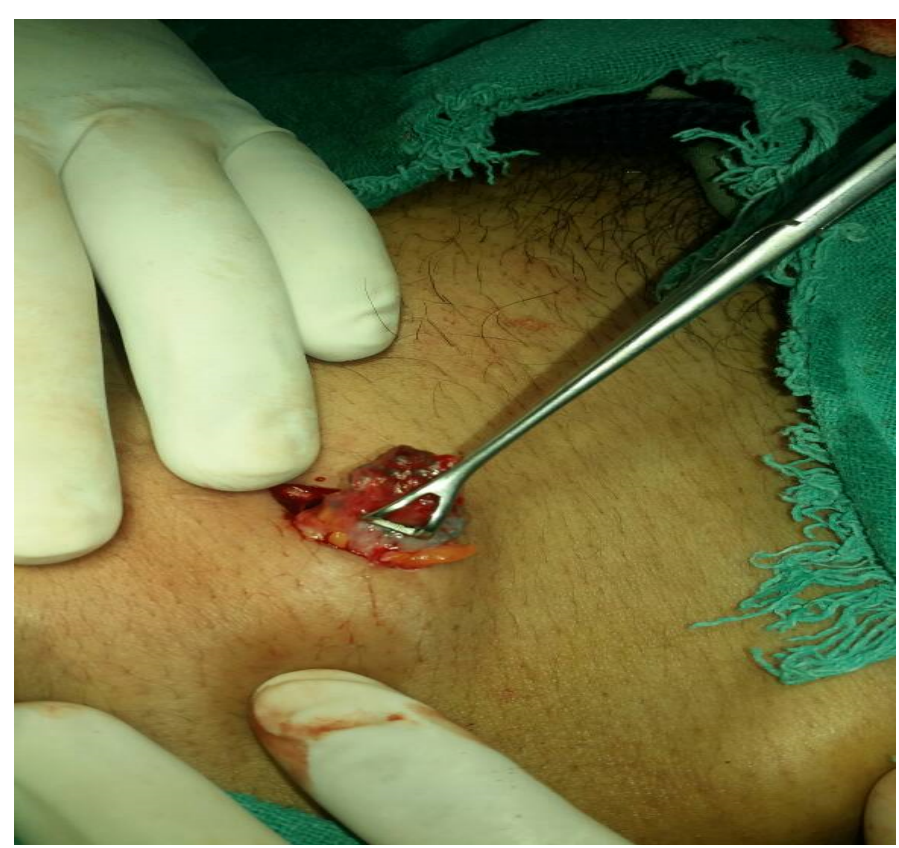

Figure 8. A bunch of pellets in parietal wall getting removed.

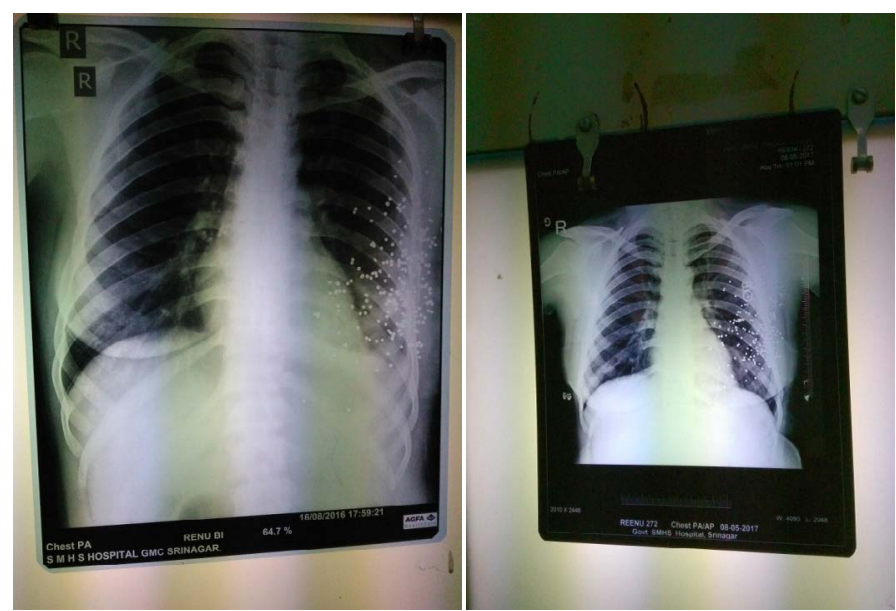

Figure 9. Radiographs of a patient showing no difference in the appearance and location of pellets after a follow up of 1 year.

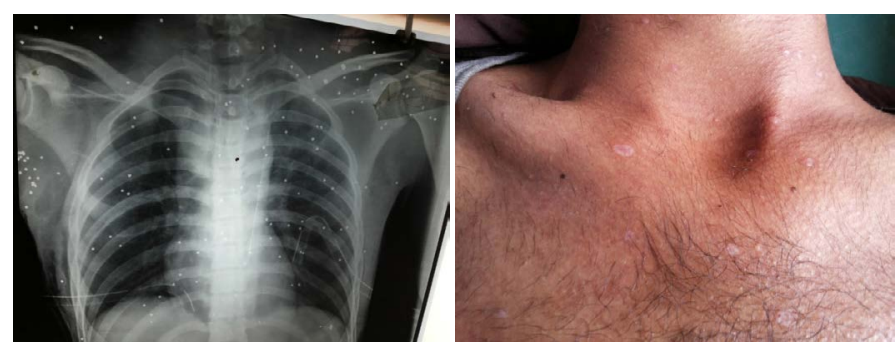

Figure 10. Pellets in anterior chest wall on X-ray in a patient(right); External appearance of the pellet wounds at 6 months follow up(Left).

any change in their form. The pellets need not to be removed from the body unless they produce such a distressing symptom that disturbs the day to day activity of an individual. There is no risk of lead poisoning for upto one year by the pellets inside human body. Mild local pain can be well treated by oral low dose analgesics and methylcobalamine and gabapentin therapy.

So, it can be concluded that pellet injured patients need to be kept in follow-up till they become symptom free and the pellets can be removed only if their presence disturbs with the daily activities of an individual. However, further long tem follow up studies are needed to ascertain the prolonged effects of pellets on serum lead levels inside human body.

\section{References}

1. Jim H (2014) Hunting with airguns. Crosman. Retrieved 10 December 2014.

2. Thoresby FP, Darlow HM (1967) The mechanisms of primary infection of bullet wounds. Br J Surg 54: 359-361.

3. Mushtaque M, Mir MF, Bhat M, Parray FQ, Khanday SA, et al. (2012) Pellet gunfire injuries among agitated mobs in Kashmir. Turkish Journal of Trauma \& Emergency Surgery 18: 255-259.

Copyright: (C2017 Mushtaq A. This is an open-access article distributed under the terms of the Creative Commons Attribution License, which permits unrestricted use, distribution, and reproduction in any medium, provided the original author and source are credited. 